\title{
On Spatial Evolution of the Solution of a Nonstandard Problem in Linear Thermo-Microstretch Elasticity
}

\author{
Emilian Bulgariu \\ Faculty of Mathematics, Alexandra Ioan Cuza University, 700506 Iasi, Romania \\ Correspondence should be addressed to Emilian Bulgariu, bulgariu.emilian@gmail.com
}

Received 3 May 2011; Accepted 17 June 2011

Academic Editor: Angelo Luongo

Copyright (c) 2011 Emilian Bulgariu. This is an open access article distributed under the Creative Commons Attribution License, which permits unrestricted use, distribution, and reproduction in any medium, provided the original work is properly cited.

An anisotropic and nonhomogeneous compressible linear thermo-microstretch elastic cylinder is subject to zero body loads and heat supply and zero lateral specific boundary conditions. The motion is induced by a time-dependent displacement, microrotation, microstretch, and temperature variation specified pointwise over the base. Further, the motion is constrained such that the displacement, microrotation, microstretch and temperature variation and their derivatives with respect to time at points in the cylinder and at a prescribed time are given in proportion to, but not identical with, their respective initial values. Two different cases for these proportional constants are treated. It is shown that certain integrals of the solution spatially evolve with respect to the axial variable. Conditions are derived that show that the integrals exhibit alternative behavior and in particular for the semi-infinite cylinder that there is either at least exponential growth or at most exponential decay.

\section{Introduction}

The theory of micromorphic bodies was introduced by Eringen $[1,2]$ in order to adequately describe the behavior of materials that have internal structure, such as liquid crystals, blood flow, polymeric substances, and porous materials. Deformation of particles that compose the material contributes to the macroscopic behavior of the body. Eringen also developed the theory of microstretch elastic solids [3] which is a generalization of the micropolar theory [4]. The particles of the solid with microstretch can expand and contract independent of translations and the rotations which they execute. Later, Eringen developed the theory of thermomicrostretch elastic solids [5]. For this class of materials, De Cicco and Nappa [6] derived the equations of the linear theory of thermo-microstretch elastic solids with the help of an entropy production inequality proposed by Green and Laws [7]. Bofill and Quintanilla [8] 
studied existence and uniqueness results. In the case of semi-infinite cylinders with the boundary lateral surface at null temperature, Quintanilla [9] established a spatial decay estimate controlled by an exponential of a polynomial of second degree. The spatial and temporal behavior of thermoelastodynamic processes for microstretch continuum materials was studied by Ciarletta and Scalia [10].

The class of the nonstandard problems attracted the attention of many researchers in the last two decades: Ames, Payne, Knops, Song, Ciarletta, Chiriţă, Quintanilla, Straughan, Passarella, and others. Knops and Payne [11] studied spatial behavior for the motion of a (semi-infinite) cylinder composed of a nonhomogeneous anisotropic linear elastic material and subject to zero body force and zero lateral boundary conditions. The initial displacement and velocity are not prescribed, nor is the asymptotic behavior at large axial distance. It is prescribed a proportion between displacement and velocity at a given time and, respectively, their initial values. Similar problems were studied by Chiriţă and Ciarletta [12] for the theory of linear thermoelasticity without energy dissipation and by Bulgariu [13] for the theory of elasticity with voids.

We consider a cylinder occupied by an anisotropic nonhomogeneous compressible linear thermo-microstretch elastic material, which is subject to null supply terms and null lateral boundary conditions. The internal energy density per unit of initial volume is assumed to be positive definite, and the constitutive coefficients are assumed bounded from above. Initial data are not prescribed, neither is the asymptotic behavior at large axial distance. We establish decay and growth exponential estimates with respect to axial variable for an integral of cross-sectional energy.

The problem studied in this paper finds application in geology and structural engineering. In [11] we have the example of a pile driven into a rigid foundation that prevents movement of the lateral boundary. The time-dependent displacement, microrotation, microstretch, and variation of temperature prescribed over the excited end constrains the motion such that the displacement, microrotation, microstretch, and variation of temperature and their derivatives with respect to time at points in the cylinder and at a prescribed time are given in proportion to, but not identical with, their respective initial values. It is desired to predict the deformation at each cross-section of the pile in terms of the base displacement, microrotation, microstretch, and variation of temperature.

\section{Notation and Basic Formulation}

Consider a prismatic cylinder $\Omega \subset \mathbb{R}^{3}$ whose bounded uniform cross-section $D \subset \mathbb{R}^{2}$ has piecewise continuously differentiable boundary $\partial D$.

The standard convention of summation over repeated suffixes is adopted, and a subscript comma denotes the spatial partial differentiation with respect to the corresponding cartesian coordinate and a superposed dot denotes differentiation with respect to time. Greek subscripts vary over $\{1,2\}$, and Latin subscripts vary over $\{1,2,3\}$. The letter $s$ is reserved for use as a time integration variable.

With respect to the chosen Cartesian coordinates, a partial volume of the cylinder will be denoted by

$$
\Omega\left(z_{1}, z_{2}\right)=\left\{\mathbf{x} \in \Omega: z_{1} \leq x_{3} \leq z_{2}\right\}
$$


and, for semi-infinite cylinder, it is convenient to introduce the abbreviations

$$
\Omega(z)=\left\{\mathbf{x} \in \Omega: z \leq x_{3}\right\}, \quad \Omega_{0}(z)=\left\{\mathbf{x} \in \Omega: z \leq x_{3} \text { at time } t=0\right\} .
$$

For the cylinder of finite length $L$ (if the cylinder is semi-finite we take $L=\infty$ ), $\Omega$ is equivalent with $\Omega(0, L)$, while $\Omega$ and $\Omega(0)$ are equivalent for the semi-infinite cylinder. To be more explicit, we will employ the notation $D\left(x_{3}, t\right)$ to indicate that respective quantities are to be evaluated at time $t$ over the cross-section whose distance from the origin is $x_{3}$.

In this paper, we consider the theory of thermo-microstretch elastic solids. The equations of this theory are [5]

(i) the evolutive equations:

$$
\begin{gathered}
t_{j i, j}+f_{i}=\rho \ddot{u}_{i}, \\
m_{j i, j}+\varepsilon_{i r s} t_{r s}+g_{i}=I_{i j} \ddot{\varphi}_{j}, \\
\pi_{i, i}-\sigma+h=J \ddot{\psi}, \\
\rho T_{0} \dot{\eta}=q_{i, i}+s \quad \text { in } \Omega \times(0, T),
\end{gathered}
$$

(ii) the constitutive equations:

$$
\begin{gathered}
t_{i j}=A_{i j r s} e_{r s}+B_{i j r s} \kappa_{r s}+D_{i j r} \gamma_{r}+A_{i j} \psi-\beta_{i j} \theta, \\
m_{i j}=B_{r s i j} e_{r s}+C_{i j r s} \kappa_{r s}+E_{i j r} \gamma_{r}+B_{i j} \psi-C_{i j} \theta, \\
3 \pi_{i}=D_{r s i} e_{r s}+E_{r s i} \kappa_{r s}+D_{i j} \gamma_{j}+d_{i} \psi-\xi_{i} \theta, \\
3 \sigma=A_{r s} e_{r s}+B_{r s} \kappa_{r s}+d_{i} \gamma_{i}+m \psi-\zeta \theta, \\
\rho \eta=\beta_{r s} e_{r s}+C_{r s} \kappa_{r s}+\xi_{i} \gamma_{i}+\zeta \psi+a \theta, \\
q_{i}=k_{i j} \theta_{, j} \quad \text { in } \bar{\Omega} \times[0, T),
\end{gathered}
$$

(iii) the geometric relations

$$
e_{i j}=u_{j, i}+\varepsilon_{j i k} \varphi_{k}, \quad \kappa_{i j}=\varphi_{j, i}, \quad \gamma_{i}=\psi_{, i}, \quad \text { on } \bar{\Omega} .
$$

In the above equations we have used the following notations: $t_{i j}$ is the stress tensor, $m_{i j}$ is the couple stress tensor, $\pi_{i}$ is the microstress vector, $\sigma$ is the scalar microstress function, $\eta$ is the specific entropy, $\rho$ is the mass density (mass in the reference configuration), $f_{i}$ is the body force, $g_{i}$ is the body couple, $h$ is the (scalar) body load, $s$ is the heat source density, $q_{i}$ is the heat flux vector, $I_{i j}$ is the microinertia tensor, $J$ is the microstretch inertia, and $\varepsilon_{i j k}$ is the alternating symbol. The variables of this theory are as follows: $u_{i}$ the components of the displacement vector, $\varphi_{i}$ the components of the microrotation vector, $\psi$ the microstretch 
function, and $\theta$ the variation of temperature from the uniform reference absolute temperature $T_{0}$.

The constitutive coefficients and $I_{i j}$ are prescribed functions of the spatial variable with the following symmetries:

$$
A_{i j r s}=A_{r s i j}, \quad C_{i j r s}=C_{r s i j}, \quad D_{i j}=D_{j i}, \quad k_{i j}=k_{j \mathrm{i}}, \quad I_{i j}=I_{j i} .
$$

Moreover we have

$$
k_{i j} \theta_{, i} \theta_{, j} \geq 0, \quad I_{i j} \varphi_{, i} \varphi_{, j} \geq 0 .
$$

We assume that $\rho, I_{i j}, J$ and the constitutive coefficients are continuous and bounded fields on the closure $\bar{\Omega}$. We also assume that the constitutive coefficients are continuous differentiable functions on $\bar{\Omega}$ and

$$
\rho(\mathbf{x}) \geq \rho_{0}>0, \quad a(\mathbf{x}) \geq a_{0}>0, \quad J(\mathbf{x}) \geq J_{0}>0, \quad I(\mathbf{x}) \geq I_{0}>0,
$$

where $I(\mathbf{x})$ denote the minimum eigenvalue of $I_{i j}(\mathbf{x})$ and $\rho_{0}, a_{0}, J_{0}, I_{0}$ are constants.

By taking into account that $k_{i j}$ is a positive definite tensor, we have

$$
k_{m} \theta_{, i} \theta_{, i} \leq k_{i j} \theta_{, i} \theta_{, j} \leq k_{M} \theta_{, i} \theta_{, i}
$$

where $k_{m}$ and $k_{M}$ are related to the minimum and the maximum eigenvalue (conductivity moduli) for $k_{i j}$. By using the Schwarz's inequality we have

$$
q_{i} q_{i} \leq k_{M} k_{i j} \theta_{, i} \theta_{, j}
$$

In what follows we denote with $P$ the nonstandard problem structured by equations (2.3)-(2.5) with null supply terms, supplemented by the lateral boundary conditions

$$
\begin{array}{ll}
\dot{u}_{i} t_{\alpha i} n_{\alpha}=0, & \dot{\varphi}_{i} m_{\alpha i} n_{\alpha}=0, \quad \dot{\psi} \pi_{\alpha} n_{\alpha}=0, \\
\theta q_{\alpha} n_{\alpha}=0, & (\mathbf{x}, t) \in(\partial D \times[0, L]) \times[0, T],
\end{array}
$$

conditions on the base

$$
\begin{gathered}
u_{i}(\mathbf{x}, t)=a_{i}\left(x_{\alpha}, t\right), \quad \varphi_{i}(\mathbf{x}, t)=b_{i}\left(x_{\alpha}, t\right), \quad \psi(\mathbf{x}, t)=c\left(x_{\alpha}, t\right), \\
\theta(\mathbf{x}, t)=\tau\left(x_{\alpha}, t\right), \quad(\mathbf{x}, t) \in D(0) \times[0, T],
\end{gathered}
$$


and the final values at time $T$ of the displacement, microrotation, microstretch, and variation of temperature and their derivatives with respect to time are proportional to their initial values, that is,

$$
\begin{gathered}
u_{i}(\mathbf{x}, T)=\lambda u_{i}(\mathbf{x}, 0), \quad \varphi_{i}(\mathbf{x}, T)=\lambda \varphi_{i}(\mathbf{x}, 0), \quad \psi(\mathbf{x}, T)=\lambda \psi(\mathbf{x}, 0), \\
\theta(\mathbf{x}, T)=v \theta(\mathbf{x}, 0) \quad \text { for } \mathbf{x} \in \Omega, \\
\dot{u}_{i}(\mathbf{x}, T)=\alpha \dot{u}_{i}(\mathbf{x}, 0), \quad \dot{\varphi}_{i}(\mathbf{x}, T)=\mu \dot{\varphi}_{i}(\mathbf{x}, 0), \quad \dot{\psi}(\mathbf{x}, T)=\beta \dot{\psi}(\mathbf{x}, 0), \quad \mathbf{x} \in \Omega,
\end{gathered}
$$

where $\mathbf{n}$ is the unit outward normal on $\partial D, a_{i}\left(x_{\alpha}, t\right), a_{i}\left(x_{\alpha}, t\right), c\left(x_{\alpha}, t\right)$, and $\tau\left(x_{\alpha}, t\right)$ are prescribed differentiable functions compatible with the initial/final data and the lateral boundary data. The constants $\alpha, \mu, \lambda, \beta$, and $v$ are prescribed and satisfy the conditions

$$
|\alpha|>1, \quad|\mu|>1, \quad|\beta|>1, \quad|\lambda|>1 \quad|\nu|>1 .
$$

The initial displacement, microrotation, microstretch, and variation of temperature and their derivatives with respect to time at points in the cylinder are not prescribed. The conditions specified on the end $D(L)$ for a finite cylinder, or at asymptotically large axial distance for the semi-infinite cylinder, are also not prescribed.

We will use the notations $\varkappa=\left\{\psi_{i j}, X_{i j}, v_{i}, \phi\right\}$ and $\varkappa^{(\alpha)}=\left\{\psi_{i j}^{(\alpha)}, x_{i j}^{(\alpha)}, v_{i}^{(\alpha)}, \phi^{(\alpha)}\right\}, \alpha=1,2$. We assume that the internal energy density per unit of volume is a positive definite quadratic form, therefore we can write

$$
\begin{aligned}
& \left.\aleph_{m} \psi_{i j} \psi_{i j}+\beth_{m} \chi_{i j} X_{i j}+\beth_{m} v_{i} v_{i}+\right\rceil_{m} \phi^{2} \\
& \left.\quad \leq 2 W(\varkappa) \leq \aleph_{M} \psi_{i j} \psi_{i j}+\beth_{M} X_{i j} X_{i j}+\beth_{M} v_{i} v_{i}+\right\rceil_{M} \phi^{2}, \text { for all } \varkappa,
\end{aligned}
$$

where $\left.\left.\aleph_{m}, \aleph_{M}, \beth_{m}, \beth_{M}, \beth_{m}, \beth_{M},\right\rceil_{m},\right\rceil_{M}$ are positive constants related to the minimum and, respectively, maximum eigenvalues of the positive definite quadratic form

$$
\begin{aligned}
2 W(\varkappa)= & A_{i j r s} \psi_{i j} \psi_{r s}+C_{i j r s} \chi_{i j} \chi_{r s}+D_{i j} v_{i} v_{j}+m \phi^{2}+2 B_{i j r s} \psi_{i j} \chi_{r s} \\
& +2 D_{i j r} \psi_{i j} v_{r}+2 E_{i j r} \chi_{i j} v_{r}+2 A_{i j} \psi_{i j} \phi+2 B_{i j} \chi_{i j} \phi+2 d_{i} v_{i} \phi .
\end{aligned}
$$

By dedublation of this quadratic form we have

$$
\begin{aligned}
2 \varepsilon\left(\varkappa^{(1)}, \varkappa^{(2)}\right)= & A_{i j r s} \psi_{i j}^{(1)} \psi_{r s}^{(2)}+C_{i j r s} \chi_{i j}^{(1)} \chi_{r s}^{(2)}+D_{i j} v_{i}^{(1)} v_{j}^{(2)}+m \phi^{(1)} \phi^{(2)} \\
& +B_{i j r s}\left[\psi_{i j}^{(1)} \chi_{r s}^{(2)}+\psi_{i j}^{(2)} \chi_{r s}^{(1)}\right]+D_{i j r}\left[\psi_{i j}^{(1)} v_{r}^{(2)}+\psi_{i j}^{(2)} v_{r}^{(1)}\right] \\
& +E_{i j r}\left[\chi_{i j}^{(1)} v_{r}^{(2)}+\chi_{i j}^{(2)} v_{r}^{(1)}\right]+A_{i j}\left[\psi_{i j}^{(1)} \phi^{(2)}+\psi_{i j}^{(2)} \phi^{(1)}\right] \\
& +B_{i j}\left[x_{i j}^{(1)} \phi^{(2)}+x_{i j}^{(2)} \phi^{(1)}\right]+d_{i}\left[v_{i}^{(1)} \phi^{(2)}+v_{i}^{(2)} \phi^{(1)}\right] .
\end{aligned}
$$


We can remark that $\varepsilon(\varkappa, \varkappa)=W(\varkappa)$. By the Cauchy-Schwarz inequality, we have

$$
\varepsilon\left(\varkappa^{(1)}, \varkappa^{(2)}\right) \leq\left[W\left(\varkappa^{(1)}\right)\right]^{1 / 2}\left[W\left(\varkappa^{(2)}\right)\right]^{1 / 2} .
$$

We introduce the following notations: $T_{i j}=t_{i j}+\beta_{i j} \theta, M_{i j}=m_{i j}+C_{i j} \theta, \Pi_{i}=3 \pi_{i}+\xi_{i} \theta$, $\Sigma=3 \sigma+\zeta \theta$. If, in (2.16), we take $\bar{\varkappa}=\left\{\left(1 / \rho_{0}\right) T_{i j},\left(1 / I_{0}\right) M_{i j},\left(1 / J_{0}\right) \Pi_{i},\left(1 / \rho_{0}\right) \Sigma\right\}$, we obtain

$$
\begin{aligned}
2 W(\bar{\varkappa}) & \leq \frac{\aleph_{M}}{\rho_{0}^{2}} T_{i j} T_{i j}+\frac{\beth_{M}}{I_{0}^{2}} M_{i j} M_{i j}+\frac{\beth_{M}}{J_{0}^{2}} \Pi_{i} \Pi_{i}+\frac{\rceil_{M}}{\rho_{0}^{2}} \Sigma^{2} \\
& \leq \varpi\left(\frac{1}{\rho_{0}} T_{i j} T_{i j}+\frac{1}{I_{0}} M_{i j} M_{i j}+\frac{1}{J_{0}} \Pi_{i} \Pi_{i}+\frac{1}{\rho_{0}} \Sigma^{2}\right),
\end{aligned}
$$

where

$$
\varpi=\max \left(\frac{\aleph_{M}}{\rho_{0}}, \frac{\beth_{M}}{I_{0}}, \frac{\beth_{M}}{J_{0}}, \frac{\urcorner_{M}}{\rho_{0}}\right)
$$

From (2.17)-(2.20), we have

$$
\begin{aligned}
\frac{1}{\rho_{0}} T_{i j} T_{i j}+\frac{1}{I_{0}} M_{i j} M_{i j}+\frac{1}{J_{0}} \Pi_{i} \Pi_{i}+\frac{1}{\rho_{0}} \Sigma^{2} & =2 \varepsilon\left(\left\{e_{i j}, \kappa_{i j}, \gamma_{i}, \psi\right\},\left\{\frac{1}{\rho_{0}} T_{i j}, \frac{1}{I_{0}} M_{i j}, \frac{1}{J_{0}} \Pi_{i}, \frac{1}{\rho_{0}} \Sigma\right\}\right) \\
\leq & {[2 W]^{1 / 2}[2 W(\bar{\varkappa})]^{1 / 2} } \\
\leq & {\left[2 \varpi W\left(\frac{1}{\rho_{0}} T_{i j} T_{i j}+\frac{1}{I_{0}} M_{i j} M_{i j}+\frac{1}{J_{0}} \Pi_{i} \Pi_{i}+\frac{1}{\rho_{0}} \Sigma^{2}\right)\right]^{1 / 2}, }
\end{aligned}
$$

where $W=W\left(\left\{e_{i j}, \kappa_{i j}, \gamma_{i}, \psi\right\}\right)$, and consequently we obtain

$$
\frac{1}{\rho_{0}} T_{i j} T_{i j}+\frac{1}{I_{0}} M_{i j} M_{i j}+\frac{1}{J_{0}} \Pi_{i} \Pi_{i} \leq 2 \varpi W
$$

By using relations (2.4), (2.5), and (2.17), we obtain

$$
t_{i j} \dot{e}_{i j}+m_{i j} \dot{\kappa}_{i j}+3 \pi_{i} \dot{\gamma}_{i}+3 \sigma \dot{\psi}+\rho \dot{\eta} \theta+\frac{1}{T_{0}} q_{i} \theta_{, i}=\dot{W}+\frac{1}{T_{0}} k_{i j} \theta_{, i} \theta_{, j}+a \theta \dot{\theta}
$$

\section{A Differential Inequality}

The aim of this section is to obtain a differential inequality for an appropriate function related to the cross-sectional energy flux. 
We introduce the following function:

$$
I\left(x_{3}\right)=\int_{0}^{T} \int_{D\left(x_{3}, s\right)} e^{-\omega s}\left(t_{3 i} \dot{u}_{i}+m_{3 i} \dot{\varphi}_{i}+3 \pi_{3} \dot{\psi}+\frac{1}{T_{0}} q_{3} \theta\right) d a d s, \quad 0 \leq x_{3} \leq L
$$

where $\omega$ is a positive parameter at our disposal whose values will be defined later.

By direct differentiation with respect to $x_{3}$ in (3.1) and by using the evolutive equations (2.3) with null supply terms, we obtain

$$
\begin{aligned}
\frac{d I}{d x_{3}}\left(x_{3}\right)=\int_{0}^{T} \int_{D\left(x_{3}, s\right)} e^{-\omega s}\left(\rho \dot{u}_{i} \ddot{u}_{i}+I_{i j} \dot{\varphi}_{i} \ddot{\varphi}_{j}+3 J \dot{\psi} \ddot{\varphi}+\rho \dot{\eta} \theta\right. \\
-t_{\alpha i, \alpha} \dot{u}_{i}+t_{3 i} \dot{u}_{i, 3}-m_{\alpha i, \alpha} \dot{\varphi}_{i}-\varepsilon_{j i k} t_{j i} \dot{\varphi}_{k}+m_{3 i} \dot{\varphi}_{i, 3} \\
\\
\left.-3 \pi_{\alpha, \alpha} \dot{\psi}+3 \sigma \dot{\psi}+3 \pi_{3} \dot{\psi}, 3-\frac{1}{T_{0}} q_{\alpha, \alpha} \theta+\frac{1}{T_{0}} q_{3} \theta_{, 3}\right) d a d s,
\end{aligned}
$$

and by using the geometric relations (2.5), we have

$$
\begin{gathered}
\frac{d I}{d x_{3}}\left(x_{3}\right)=\int_{0}^{T} \int_{D\left(x_{3}, s\right)} e^{-\omega s}\left(\rho \dot{u}_{i} \ddot{u}_{i}+I_{i j} \dot{\varphi}_{i} \ddot{\varphi}_{j}+3 J \dot{\psi} \ddot{\varphi}+\rho \dot{\eta} \theta\right. \\
\left.+t_{i j} \dot{e}_{i j}+m_{i j} \dot{\kappa}_{i j}+3 \pi_{i} \dot{\gamma}_{i}+3 \sigma \dot{\psi}+\frac{1}{T_{0}} q_{i} \theta, i\right) d a d s \\
-\int_{0}^{T} \int_{D\left(x_{3}, s\right)} e^{-\omega s}\left(t_{\alpha i} \dot{u}_{i}+m_{\alpha i} \dot{\varphi}_{i}+3 \pi_{\alpha} \dot{\psi}+\frac{1}{T_{0}} q_{\alpha} \theta\right)_{, \alpha} d a d s .
\end{gathered}
$$

The divergence theorem and the lateral boundary conditions (2.11) ensure us that the final integral in the right-hand term vanishes. Using relation (2.24), we deduce

$$
\begin{aligned}
\frac{d I}{d x_{3}}\left(x_{3}\right)= & \frac{1}{2} \int_{0}^{T} \int_{D\left(x_{3}, s\right)} e^{-\omega s} \frac{\partial}{\partial s}\left(\rho \dot{u}_{i} \dot{u}_{i}+I_{i j} \dot{\varphi}_{i} \dot{\varphi}_{j}+3 J \dot{\psi} \dot{\psi} \dot{\psi}+2 W+a \theta^{2}\right) d a d s \\
& +\int_{0}^{T} \int_{D\left(x_{3}, s\right)} \frac{1}{T_{0}} e^{-\omega s} k_{i j} \theta_{, i} \theta_{, j} d a d s .
\end{aligned}
$$

Finally, the above equation yields

$$
\begin{aligned}
\frac{d I}{d x_{3}}\left(x_{3}\right)= & E\left(x_{3}, T\right)-E\left(x_{3}, 0\right)+\int_{0}^{T} \omega E\left(x_{3}, s\right) d s \\
& +\int_{0}^{T} \int_{D\left(x_{3}, s\right)} \frac{1}{T_{0}} e^{-\omega s} k_{i j} \theta_{, i} \theta_{, j} d a d s,
\end{aligned}
$$


where

$$
E\left(x_{3}, t\right)=\frac{1}{2} \int_{D\left(x_{3}, s\right)} e^{-\omega s}\left(\rho \dot{u}_{i} \dot{u}_{i}+I_{i j} \dot{\varphi}_{i} \dot{\varphi}_{j}+3 J \dot{\psi}^{2}+2 W+a \theta^{2}\right) d a, \quad t \in[0, T] .
$$

Therefore, by means of relations (2.13) and (2.14), we have

$$
\begin{aligned}
\frac{d I}{d x_{3}}\left(x_{3}\right)= & \frac{1}{2}\left(e^{-\omega T} \alpha^{2}-1\right) \int_{D\left(x_{3}, 0\right)} \rho \dot{u}_{i} \dot{u}_{i} d a \\
& +\frac{1}{2}\left(e^{-\omega T} \mu^{2}-1\right) \int_{D\left(x_{3}, 0\right)} I_{i j} \dot{\varphi}_{i} \dot{\varphi}_{j} d a \\
& +\frac{1}{2}\left(e^{-\omega T} \beta^{2}-1\right) \int_{D\left(x_{3}, 0\right)} 3 J \dot{\psi}^{2} d a \\
& +\frac{1}{2}\left(e^{-\omega T} \lambda^{2}-1\right) \int_{D\left(x_{3}, 0\right)} 2 W d a \\
& +\frac{1}{2}\left(e^{-\omega T} v^{2}-1\right) \int_{D\left(x_{3}, 0\right)} a \theta^{2} d a \\
& +\int_{0}^{T} \omega E\left(x_{3}, s\right) d s+\int_{0}^{T} \int_{D\left(x_{3}, s\right)} \frac{1}{T_{0}} e^{-\omega s} k_{i j} \theta_{, i} \theta_{, j} d a d s .
\end{aligned}
$$

We will choose the parameter $\omega$ so that we have

$$
0<\chi_{\omega}=\frac{1}{2} \min \left(e^{-\omega T} \alpha^{2}-1, e^{-\omega T} \mu^{2}-1, e^{-\omega T} \beta^{2}-1, e^{-\omega T} \lambda^{2}-1, e^{-\omega T} v^{2}-1\right)
$$

assumming that $\omega$ ranges in the set

$$
0<\omega<\frac{2}{T} \min (\ln |\alpha|, \ln |\mu|, \ln |\beta|, \ln |\lambda|, \ln |v|)
$$

if we suppose that conditions (2.15) hold true.

In this context, we note that

$$
\begin{aligned}
\frac{d I}{d x_{3}}\left(x_{3}\right) \geq & \chi_{\omega} \int_{D\left(x_{3}, 0\right)}\left(\rho \dot{u}_{i} \dot{u}_{i}+I_{i j} \dot{\varphi}_{i} \dot{\varphi}_{j}+3 J \dot{\varphi}^{2}+2 W+a \theta^{2}\right) d a \\
& +\int_{0}^{T} \omega E\left(x_{3}, s\right) d s+\int_{0}^{T} \int_{D\left(x_{3}, s\right)} \frac{1}{T_{0}} e^{-\omega s} k_{i j} \theta_{, i} \theta_{, j} d a d s \geq 0
\end{aligned}
$$

in view of assumptions of the positive definitiveness of $W$ and $k_{i j}$. Therefore, we can conclude that $I\left(x_{3}\right)$ is a nondecreasing function with respect to $x_{3}$ on $[0, L]$. 
Next, we want to obtain an appropriate estimate for the function $I\left(x_{3}\right)$. Using the constitutive equations, the Schwarz's inequality, the arithmetic-mean inequality, and relation (2.23), we obtain the inequality

$$
\begin{aligned}
\frac{1}{\rho_{0}} t_{i j} t_{i j}+\frac{1}{I_{0}} m_{i j} m_{i j}+\frac{1}{J_{0}} 3 \pi_{i} \pi_{i}= & \left(\frac{T_{i j}}{\sqrt{\rho_{0}}}-\frac{\beta_{i j}}{\sqrt{\rho_{0}}} \theta\right)\left(\frac{T_{i j}}{\sqrt{\rho_{0}}}-\frac{\beta_{i j}}{\sqrt{\rho_{0}}} \theta\right) \\
& +\left(\frac{M_{i j}}{\sqrt{I_{0}}}-\frac{C_{i j}}{\sqrt{I_{0}}} \theta\right)\left(\frac{M_{i j}}{\sqrt{I_{0}}}-\frac{C_{i j}}{\sqrt{I_{0}}} \theta\right) \\
& +\left(\frac{\Pi_{i}}{\sqrt{3 J_{0}}}-\frac{\xi_{i}}{\sqrt{3 J_{0}}} \theta\right)\left(\frac{\Pi_{i}}{\sqrt{3 J_{0}}}-\frac{\xi_{i}}{\sqrt{3 J_{0}}} \theta\right) \\
\leq & \left(1+\varepsilon_{1}\right)\left(\frac{1}{\rho_{0}} T_{i j} T_{i j}+\frac{1}{I_{0}} M_{i j} M_{i j}+\frac{1}{3 J_{0}} \Pi_{i} \Pi_{i}\right) \\
& +\left(1+\frac{1}{\varepsilon_{1}}\right)\left(\frac{1}{\rho_{0}} \beta_{i j} \beta_{i j}+\frac{1}{I_{0}} C_{i j} C_{i j}+\frac{1}{3 J_{0}} \xi_{i} \xi_{i}\right) \theta^{2} \\
\leq & \left(1+\varepsilon_{1}\right) 2 \varpi W+\left(1+\frac{1}{\varepsilon_{1}}\right) M^{2} \theta^{2}, \quad \forall \varepsilon_{1}>0,
\end{aligned}
$$

where

$$
M^{2}=\max _{\bar{\Omega}}\left(\frac{1}{\rho_{0}} \beta_{i j} \beta_{i j}+\frac{1}{I_{0}} C_{i j} C_{i j}+\frac{1}{3 J_{0}} \xi_{i} \xi_{i}\right)
$$

Using Schwarz's inequality, arithmetic-mean inequality, and relations (2.10) and (3.11), we obtain the estimate

$$
\begin{aligned}
&\left|I\left(x_{3}\right)\right| \leq \int_{0}^{T} \int_{D\left(x_{3}, s\right)} e^{-\omega s}\{ \frac{\varepsilon_{2}}{2}\left[\frac{1}{\rho_{0}} t_{3 i} t_{3 i}+\frac{1}{I_{0}} m_{3 i} m_{3 i}+\frac{3}{J_{0}} \pi_{3} \pi_{3}\right]+\frac{\varepsilon_{3}}{2 T_{0} a_{0}} q_{3} q_{3} \\
&\left.+\frac{1}{2 \varepsilon_{2}}\left[\rho \dot{u}_{i} \dot{u}_{i}+I_{i j} \dot{\varphi}_{i} \dot{\varphi}_{j}+3 J \dot{\psi}^{2}\right]+\frac{1}{2 T_{0} \varepsilon_{3}} a \theta^{2}\right\} d a d s \\
& \leq \int_{0}^{T} \int_{D\left(x_{3}, s\right)} e^{-\omega s}\left\{\frac{\varepsilon_{2}}{2}\left[\frac{1}{\rho_{0}} t_{i j} t_{i j}+\frac{1}{I_{0}} m_{i j} m_{i j}+\frac{3}{J_{0}} \pi_{i} \pi_{i}\right]+\frac{\varepsilon_{3}}{2 T_{0} a_{0}} q_{i} q_{i}\right. \\
&+\left.\frac{1}{2 \varepsilon_{2}}\left[\rho \dot{u}_{i} \dot{u}_{i}+I_{i j} \dot{\varphi}_{i} \dot{\varphi}_{j}+3 J \dot{\psi}^{2}\right]+\frac{1}{2 T_{0} \varepsilon_{3}} a \theta^{2}\right\} d a d s \\
& \leq \int_{0}^{T} \int_{D\left(x_{3}, s\right)} e^{-\omega s}\left\{\frac{1}{\omega \varepsilon_{2}} \frac{\omega}{2}\left[\rho \dot{u}_{i} \dot{u}_{i}+I_{i j} \dot{\varphi}_{i} \dot{\varphi}_{j}+3 J \dot{\psi}^{2}\right]\right. \\
&+ \frac{\varepsilon_{2}\left(1+\varepsilon_{1}\right) \varpi}{\omega} \frac{\omega}{2} 2 W+\left[\frac{\varepsilon_{2} M M^{2}\left(1+\varepsilon_{1}\right)}{\omega a_{0} \varepsilon_{1}}+\frac{1}{\omega T_{0} \varepsilon_{3}}\right] \frac{\omega}{2} a \theta^{2} \\
&\left.+\frac{\varepsilon_{3} k_{M}}{2 a_{0}} \frac{1}{T_{0}} k_{i j} \theta_{, i} \theta_{, j}\right\} d a d s, \quad \forall \varepsilon_{1}, \varepsilon_{2}, \varepsilon_{3}>0 .
\end{aligned}
$$


Further, we equate the coefficients of all energetic terms in the last integral imposing that

$$
\frac{1}{\omega \varepsilon_{2}}=\frac{\varepsilon_{2}\left(1+\varepsilon_{1}\right) \varpi}{\omega}=\frac{M^{2} \varepsilon_{2}\left(1+\varepsilon_{1}\right)}{\omega a_{0} \varepsilon_{1}}+\frac{1}{\omega T_{0} \varepsilon_{3}}=\frac{\varepsilon_{3} k_{M}}{2 a_{0}},
$$

and hence we have

$$
\varepsilon_{2}=\sqrt{\frac{1}{\left(1+\varepsilon_{1}\right) \varpi}}, \quad \varepsilon_{3}=\frac{2 a_{0}}{k_{M} \omega} \sqrt{\left(1+\varepsilon_{1}\right) \varpi}
$$

where

$$
\varepsilon_{1}=\frac{1}{2}\left[-\left(1-\frac{2 T_{0} M^{2}+\omega k_{M}}{2 a_{0} T_{0} \varpi}\right)+\sqrt{\left(1-\frac{2 T_{0} M^{2}+\omega k_{M}}{2 a_{0} T_{0} \varpi}\right)^{2}+4 \frac{M^{2}}{a_{0} \varpi}}\right] .
$$

Because we imposed (3.14), we can multiply (3.13) by $c=\omega \varepsilon_{2}$ and obtain

$$
c\left|I\left(x_{3}\right)\right| \leq \int_{0}^{T} \omega E\left(x_{3}, s\right) d s+\int_{0}^{T} \int_{D\left(x_{3}, s\right)} \frac{1}{T_{0}} e^{-\omega s} k_{i j} \theta_{, i} \theta_{, j} d a d s,
$$

and from (3.10) we obtain the first-order differential inequality

$$
c\left|I\left(x_{3}\right)\right| \leq \frac{d I}{d x_{3}}\left(x_{3}\right), \quad \forall x_{3} \in[0, L] .
$$

\section{Spatial Behaviour of $I\left(x_{3}\right)$}

In this section we determine the spatial evolution of the solution of the nonstandard problem $p$ by integrating (3.18). We first consider that the cylinder has a finite length (i.e., $L<\infty)$. We have only two possibilities: (a) $I\left(x_{3}\right) \leq 0$ for all $x_{3} \in[0, L]$ or (b) there exists $z \in[0, L]$ so that $I(z)>0$. It is easy to see that, if $I(L) \leq 0$, we are in the case (a).

Let us first consider the case (a). Because $I\left(x_{3}\right)$ it is a nondecreasing function with respect to $x_{3}$ on $[0, L]$, we have

$$
\frac{d I}{d x_{3}}\left(x_{3}\right)+c I\left(x_{3}\right) \geq 0, \quad 0 \leq x_{3} \leq L
$$

which after integration leads to the following Saint-Venant-type decay estimate:

$$
0 \leq-I\left(x_{3}\right) \leq-I(0) e^{-c x_{3}}, \quad 0 \leq x_{3} \leq L
$$


For a cylinder of finite length $L$, we have to prescribe such boundary conditions on the end $x_{3}=L$ that implies $I(L)=0$, and then we will predict a spatial exponential decay as described in (4.2). inequality

For the case (b), we have $0 \leq I(z) \leq I\left(x_{3}\right)$ for $z \leq x_{3} \leq L$, and hence (3.18) implies the

$$
0 \leq \frac{d I}{d x_{3}}\left(x_{3}\right)-c I\left(x_{3}\right), \quad z \leq x_{3} \leq L
$$

which, by integrating, yields the following growth estimate

$$
I\left(x_{3}\right) \geq I(z) e^{c\left(x_{3}-z\right)}, \quad z \leq x_{3} \leq L
$$

Let us discuss further the case of a semi-infinite cylinder (i.e., the case when $L \rightarrow \infty$ ). If $I\left(x_{3}\right) \leq 0$ for all $x_{3} \in[0, \infty)$, we obtain that $I\left(x_{3}\right) \rightarrow 0$ as $x_{3} \rightarrow \infty$, and hence relation (3.10) gives the following decay estimate for the weighted total energy:

$$
\mathcal{E}\left(x_{3}\right) \leq-I(0) e^{-c x_{3}}, \quad 0 \leq x_{3}<\infty,
$$

where

$$
\begin{aligned}
\mathcal{E}\left(x_{3}^{*}\right)= & \chi_{\omega} \int_{\Omega_{0}\left(x_{3}^{*}\right)}\left(\rho \dot{u}_{i} \dot{u}_{i}+I_{i j} \dot{\varphi}_{i} \dot{\varphi}_{j}+3 J \dot{\psi}^{2}+2 W+a \theta^{2}\right) d v \\
& +\lim _{x_{3} \rightarrow \infty} \int_{0}^{T} \int_{x_{3}^{*}}^{x_{3}} \omega E(\vartheta, s) d \vartheta d s+\int_{0}^{T} \int_{\Omega\left(x_{3}^{*}\right)} \frac{1}{T_{0}} e^{-\omega s} k_{i j} \theta_{, i} \theta_{, j} d v d s, \quad 0 \leq x_{3}^{*}<\infty .
\end{aligned}
$$

If there is $z \in[0, \infty)$ so that $I(z)>0$, for the semi-infinite cylinder, $I\left(x_{3}\right)$ becomes unbounded for $x_{3} \rightarrow \infty$ and hence $\varepsilon\left(x_{3}\right)$ is infinite.

We have established a Phragmén-Lindelöf alternative type for the semi-infinite cylinder.

\section{Further Comments}

In this paper we have discussed only the case when $|\alpha|>1,|\mu|>1,|\beta|>1,|\lambda|>1$, and $|v|>1$. When we take the conditions $|\alpha|<1,|\mu|<1,|\beta|<1,|\lambda|<1$ and $|\nu|<1$ we cannot find a suitable bound because there is nonuniqueness in this case (see e.g., Quintanilla and Straughan [14] for an argument concerning this subject).

In the previous sections we have considered the nonstandard problem $D$ in which the proportionality coefficients of displacement, microrotation, microstretch, and variation of temperature with their derivatives with respect to time at the time $T$ and their respective 
initial values are given by (2.13) and (2.14). We consider a similar nonstandard problem $p^{*}$ given by (2.3)-(2.5) with null supply terms, and instead of conditions (2.13), we have

$$
\begin{gathered}
u_{i}(\mathbf{x}, T)=\lambda_{1} u_{i}(\mathbf{x}, 0), \quad \varphi_{i}(\mathbf{x}, T)=\lambda_{2} \varphi_{i}(\mathbf{x}, 0), \quad \psi(\mathbf{x}, T)=\lambda_{3} \psi(\mathbf{x}, 0), \\
\theta(\mathbf{x}, T)=v \theta(\mathbf{x}, 0), \quad \mathbf{x} \in \Omega,
\end{gathered}
$$

where $\lambda_{i}$ and $\lambda_{j}$ can be different for $i \neq j$, with conditions (2.14) remaining valid. The problem $p^{*}$ has the lateral boundary conditions

$$
\begin{gathered}
\dot{u}_{i} t_{\alpha i} n_{\alpha}=0, \quad \varphi=0, \quad \dot{\psi} \pi_{\alpha} n_{\alpha}=0, \\
\theta q_{\alpha} n_{\alpha}=0, \quad(\mathbf{x}, t) \in(\partial D \times[0, L]) \times[0, T]
\end{gathered}
$$

and the conditions on the base

$$
\begin{gathered}
u_{i}(\mathbf{x}, t)=a_{i}\left(x_{\alpha}, t\right), \quad \varphi_{i}(\mathbf{x}, t)=b_{i}\left(x_{\alpha}, t\right), \quad \psi(\mathbf{x}, t)=c\left(x_{\alpha}, t\right) \\
\theta(\mathbf{x}, t)=\tau\left(x_{\alpha}, t\right), \quad(\mathbf{x}, t) \in D(0) \times[0, \mathrm{~T}]
\end{gathered}
$$

We are interested in what conditions we would have to take for the constants $\lambda_{1}, \lambda_{2}$, and $\lambda_{3}$ so that our study given in the previous sections may follow the same path.

The internal energy density per unit of volume is a positive definite quadratic form and so, for $\varkappa=\left\{e_{i j}, \kappa_{i j}, \gamma_{i}, \psi\right\}$ in (2.16), at the moment $t=T$, we have

$$
\begin{aligned}
2 \int_{D\left(x_{3}, T\right)} W d a \geq & \aleph_{m} \int_{D\left(x_{3}, T\right)} e_{i j} e_{i j} d a+\beth_{m} \int_{D\left(x_{3}, T\right)} \kappa_{i j} \kappa_{i j} d a \\
& \left.+\beth_{m} \int_{D\left(x_{3}, T\right)} \gamma_{i} \gamma_{i} d a+\right\rceil_{m} \int_{D\left(x_{3}, T\right)} \psi^{2} d a \\
= & \aleph_{m} \int_{D\left(x_{3}, 0\right)}\left(\lambda_{1}^{2} u_{j, i} u_{j, i}+2 \lambda_{1} \lambda_{2} \varepsilon_{j i k} u_{j, i} \varphi_{k}+2 \lambda_{2}^{2} \varphi_{k} \varphi_{k}\right)^{2} d a \\
& +\frac{\beth_{m} \lambda_{2}^{2}}{2} \int_{D\left(x_{3}, 0\right)} \varphi_{j, i} \varphi_{j, i} d a+\frac{\beth_{m} \lambda_{2}^{2}}{2} \int_{D\left(x_{3}, 0\right)} \varphi_{j, i} \varphi_{j, i} d a \\
& \left.+\beth_{m} \lambda_{3}^{2} \int_{D\left(x_{3}, 0\right)} \psi_{, i} \psi_{, i} d a+\right\rceil_{m} \lambda_{3}^{2} \int_{D\left(x_{3}, 0\right)} \psi^{2} d a .
\end{aligned}
$$

The condition required in (5.2) that $\varphi=0$ on $\partial D\left(x_{3}\right)$ gives us the possibility to apply the Poincaré inequality

$$
\int_{D\left(x_{3}, 0\right)} \varphi_{j, i} \varphi_{j, i} d a \geq \delta \int_{D\left(x_{3}, 0\right)} \varphi_{i} \varphi_{i} d a
$$


with $\delta$ a positive constant. Using the arithmetic-mean inequality, we can deduce

$$
2 \lambda_{1} \lambda_{2} \varepsilon_{j i k} u_{j, i} \varphi_{k} \geq-2\left|\lambda_{1} u_{j, i}\right|\left|\lambda_{2} \varepsilon_{j i k} \varphi_{k}\right| \geq-\varepsilon \lambda_{1}^{2} u_{j, i} u_{j, i}-\frac{2}{\varepsilon} \lambda_{2}^{2} \varphi_{k} \varphi_{k}, \quad \forall \varepsilon>0 .
$$

Combining relations (5.5), (5.6), and (5.4), we have

$$
\begin{aligned}
2 \int_{D\left(x_{3}, T\right)} W d a \geq & (1-\varepsilon) \aleph_{m} \lambda_{1}^{2} \int_{D\left(x_{3}, 0\right)} u_{j, i} u_{j, i} d a \\
& +\left(2 \aleph_{m}-\frac{2 \aleph_{m}}{\varepsilon}+\frac{\beth_{m} \delta}{2}\right) \lambda_{2}^{2} \int_{D\left(x_{3}, 0\right)} \varphi_{i} \varphi_{i} d a \\
& +\frac{\beth_{m} \lambda_{2}^{2}}{2} \int_{D\left(x_{3}, 0\right)} \varphi_{j, i} \varphi_{j, i} d a+\beth_{m} \lambda_{3}^{2} \int_{D\left(x_{3}, 0\right)} \psi_{, i} \psi_{, i} d a \\
& +\rceil_{m} \lambda_{3}^{2} \int_{D\left(x_{3}, 0\right)} \psi^{2} d a .
\end{aligned}
$$

Requiring that

$$
\left(1+\frac{\beth_{m} \delta}{4 \aleph_{m}}\right)^{-1}<\varepsilon<1
$$

we ensured that the brackets from the right-hand terms in (5.7) are positive.

Using the inequality

$$
e_{i j} e_{i j}=\left(u_{j, i}+\varepsilon_{j i k} \varphi_{k}\right)^{2}<2 u_{j, i} u_{j, i}+4 \varphi_{k} \varphi_{k}
$$

from (2.16), at the moment $t=0$, we have

$$
\begin{aligned}
2 \int_{D\left(x_{3}, 0\right)} W d a \leq & \aleph_{M} \int_{D\left(x_{3}, 0\right)} e_{i j} e_{i j} d a+\beth_{M} \int_{D\left(x_{3}, 0\right)} \kappa_{i j} \kappa_{i j} d a \\
& \left.+\beth_{M} \int_{D\left(x_{3}, 0\right)} \gamma_{i} \gamma_{i} d a+\right\urcorner_{M} \int_{D\left(x_{3}, 0\right)} \psi^{2} d a \\
\leq & 2 \aleph_{M} \int_{D\left(x_{3}, 0\right)} u_{j, i} u_{j, i} d a+4 \aleph_{M} \int_{D\left(x_{3}, 0\right)} \varphi_{k} \varphi_{k} d a \\
& +\beth_{M} \int_{D\left(x_{3}, 0\right)} \varphi_{j, i} \varphi_{j, i} d a+\beth_{M} \int_{D\left(x_{3}, 0\right)} \psi_{, i} \psi_{, i} d a \\
& +\rceil_{M} \int_{D\left(x_{3}, 0\right)} \psi^{2} d a .
\end{aligned}
$$


Combining relations (5.7) and (5.10), we have

$$
\begin{aligned}
& \frac{1}{2} e^{-\omega T} \int_{D\left(x_{3}, T\right)} 2 W d a-\frac{1}{2} \int_{D\left(x_{3}, 0\right)} 2 W \\
& \geq \frac{1}{2}\left[\frac{(1-\varepsilon) e^{-\omega T} \aleph_{m} \lambda_{1}^{2}}{2 \aleph_{M}}-1\right] 2 \aleph_{M} \int_{D\left(x_{3}, 0\right)} u_{j, i} u_{j, i} d a \\
&+\frac{1}{2}\left[\frac{\left(4 \aleph_{m}-4 \aleph_{m} \varepsilon^{-1}+\beth_{m} \delta\right) e^{-\omega T} \lambda_{2}^{2}}{8 \aleph_{M}}-1\right] 4 \aleph_{M} \int_{D\left(x_{3}, 0\right)} \varphi_{i} \varphi_{i} d a \\
&+\frac{1}{2}\left[\frac{e^{-\omega T} \beth_{m} \lambda_{2}^{2}}{2 \beth_{M}}-1\right] \beth_{M} \int_{D\left(x_{3}, 0\right)} \varphi_{j, i} \varphi_{j, i} d a \\
&+\frac{1}{2}\left[\frac{e^{-\omega T} \beth_{m} \lambda_{3}^{2}}{\beth_{M}}-1\right] \beth_{M} \int_{\mathrm{D}\left(x_{3}, 0\right)} \psi_{, i} \psi_{, i} d a \\
&\left.+\frac{1}{2}\left[\frac{\left.e^{-\omega T}\right\rceil_{m} \lambda_{3}^{2}}{\beth_{M}}-1\right]\right\rceil_{m} \int_{D\left(x_{3}, 0\right)} \psi^{2} d a,
\end{aligned}
$$

and so, instead of relation (3.7), in the case of the problem $p^{*}$ we obtain

$$
\begin{aligned}
\frac{d I}{d x_{3}}\left(x_{3}\right) \geq & \frac{1}{2}\left(e^{-\omega T} \alpha^{2}-1\right) \int_{D\left(x_{3}, 0\right)} \rho \dot{u}_{i} \dot{u}_{i} d a+\frac{1}{2}\left(e^{-\omega T} \mu^{2}-1\right) \int_{D\left(x_{3}, 0\right)} I_{i j} \dot{\varphi}_{i} \dot{\varphi}_{j} d a \\
& +\frac{1}{2}\left(e^{-\omega T} \beta^{2}-1\right) \int_{D\left(x_{3}, 0\right)} 3 J \dot{\psi}^{2} d a+\frac{1}{2}\left(e^{-\omega T} v^{2}-1\right) \int_{D\left(x_{3}, 0\right)} a \theta^{2} d a \\
& +\frac{1}{2}\left[\frac{(1-\varepsilon) e^{-\omega T} \aleph_{m} \lambda_{1}^{2}}{2 \aleph_{M}}-1\right] 2 \aleph_{M} \int_{D\left(x_{3}, 0\right)} u_{j, i} u_{j, i} d a \\
& +\frac{1}{2}\left[\frac{\left(4 \aleph_{m}-4 \aleph_{m} \varepsilon^{-1}+\beth_{m} \delta\right) e^{-\omega T} \lambda_{2}^{2}}{8 \aleph_{M}}-1\right] 4 \aleph_{M} \int_{D\left(x_{3}, 0\right)} \varphi_{i} \varphi_{i} d a \\
& +\frac{1}{2}\left[\frac{e^{-\omega T} \beth_{m} \lambda_{2}^{2}}{2 \beth_{M}}-1\right] \beth_{M} \int_{D\left(x_{3}, 0\right)} \varphi_{j, i} \varphi_{j, i} d a \\
& +\frac{1}{2}\left[\frac{e^{-\omega T} \beth_{m} \lambda_{3}^{2}}{\beth_{M}}-1\right] \beth_{M} \int_{D\left(x_{3}, 0\right)} \psi_{, i} \psi_{, i} d a \\
& \left.+\frac{1}{2}\left[\frac{\left.e^{-\omega T}\right\urcorner_{m} \lambda_{3}^{2}}{\rceil_{M}}-1\right]\right\rceil_{m} \int_{D\left(x_{3}, 0\right)} \psi^{2} d a+\int_{0}^{T} \omega \mathfrak{E}\left(x_{3}, s\right) d s \\
& +\int_{0}^{T} \int_{D\left(x_{3}, s\right)} \frac{1}{T_{0}} e^{-\omega s} k_{i j} \theta_{, i} \theta_{, j} d a d s .
\end{aligned}
$$


We choose $\omega$ to ensure that

$$
\begin{aligned}
0<\chi_{\omega}=\frac{1}{2} \min ( & e^{-\omega T} \alpha^{2}-1, e^{-\omega T} \mu^{2}-1, e^{-\omega T} \beta^{2}-1, e^{-\omega T} v^{2}-1, \\
& \frac{(1-\varepsilon) e^{-\omega T} \aleph_{m} \lambda_{1}^{2}}{2 \aleph_{M}}-1, \frac{\left(4 \aleph_{m}-4 \aleph_{m} \varepsilon^{-1}+\beth_{m} \delta\right) e^{-\omega T} \lambda_{2}^{2}}{8 \aleph_{M}}-1, \\
& \left.\frac{e^{-\omega T} \beth_{m} \lambda_{2}^{2}}{2 \beth_{M}}-1, \frac{e^{-\omega T} \beth_{m} \lambda_{3}^{2}}{\beth_{M}}-1, \frac{\left.e^{-\omega T}\right\rceil_{m} \lambda_{3}^{2}}{\rceil_{M}}-1\right),
\end{aligned}
$$

and so, the range from which we can take $\omega$ is

$$
\begin{gathered}
0<\omega<\frac{1}{T} \ln \min \left(\alpha^{2}, \mu^{2}, \beta^{2}, v^{2}, \frac{(1-\varepsilon) \aleph_{m} \lambda_{1}^{2}}{2 \aleph_{M}}, \frac{\left(4 \aleph_{m}-4 \aleph_{m} \varepsilon^{-1}+\beth_{m} \delta\right) \lambda_{2}^{2}}{8 \aleph_{M}},\right. \\
\left.\frac{\beth_{m} \lambda_{2}^{2}}{2 \beth_{M}}, \frac{\beth_{m} \lambda_{3}^{2}}{\beth_{M}}, \frac{\rceil_{m} \lambda_{3}^{2}}{\rceil_{M}}\right)
\end{gathered}
$$

if the conditions $(2.15)_{1,2,3,5}$ hold true and

$$
\begin{gathered}
\left|\lambda_{1}\right|>\sqrt{\frac{2 \aleph_{M}}{\aleph_{m}}}>1, \quad\left|\lambda_{2}\right|>\max \left(\sqrt{\frac{8 \aleph_{M}}{4 \aleph_{m}+\beth_{m} \delta}}, \sqrt{\frac{2 \beth_{M}}{\beth_{m}}}\right)>1, \\
\left|\lambda_{3}\right|>\max \left(\sqrt{\frac{\beth_{M}}{\beth_{m}}}, \sqrt{\frac{\rceil_{M}}{\rceil_{m}}}\right)>1 .
\end{gathered}
$$

From relations (5.12) and (5.13), we obtain an inequality similar to (3.10) and so, we can continue like in Sections 3 and 4 to obtain a Saint-Venant type estimate or a PhragménLindelöf-type estimate.

In conclusion, if we replace conditions (2.13) with those given in (5.1), the results obtained in Sections 3 and 4 hold true if in (2.11) we change the lateral boundary condition $\dot{\varphi} m_{\alpha i} n_{\alpha}=0$ with $\varphi=0$ for $(\mathbf{x}, t) \in(\partial D \times[0, L]) \times[0, T]$ and the constants $\lambda_{1}, \lambda_{2}, \lambda_{3}, \nu, \alpha, \beta$ and $\mu$ must satisfy conditions $(2.15)_{1,2,3,5}$ and (5.15).

\section{Acknowledgment}

The author acknowledges support from the Romanian Ministry of Education and Research through CNCSIS-UEFISCSU, Project PN II-RU TE code 184, no. 86/30.07.2010.

\section{References}

[1] A. C. Eringen, "Mechanics of micromorphic materials," in Proceedings of the 2nd International Congress of Applied Mechanics, H. Gortler, Ed., pp. 131-138, Freudenstadt, Stuttgart, Germany; Springer, Berlin, Germany, 1966. 
[2] A. C. Eringen, "Mechanics of micromorphic continua," in Proceedings of the International Union of Theoretical and Applied Mechanics Symposium, E. Kroner, Ed., pp. 18-35, Springer, Berlin, Germany, 1968.

[3] A. C. Eringen, "Micropolar elastic solids with stretch," in Prof Dr Mustafa Inan Anisina, pp. 1-18, Ari Kitabevi Matbaasi, Istanbul, Turkey, 1971.

[4] A. C. Eringen, "Linear theory of micropolar elasticity," Journal of Applied Mathematics and Mechanics, vol. 15, pp. 909-923, 1966.

[5] A. C. Eringen, "Theory of thermo-microstretch elastic solids," International Journal of Engineering Science, vol. 28, no. 12, pp. 1291-1301, 1990.

[6] S. De Cicco and L. Nappa, "On the theory of thermomicrostretch elastic solids," Journal of Thermal Stresses, vol. 22, no. 6, pp. 565-580, 1999.

[7] A. E. Green and N. Laws, "On the entropy production inequality," Archive for Rational Mechanics and Analysis, vol. 45, no. 1, pp. 47-53, 1972.

[8] F. Bofill and R. Quintanilla, "Some qualitative results for the linear theory of thermo-microstretch elastic solids," International Journal of Engineering Science, vol. 33, no. 14, pp. 2115-2125, 1995.

[9] R. Quintanilla, "On the spatial decay for the dynamical problem of thermo-microstretch elastic solids," International Journal of Engineering Science, vol. 40, no. 2, pp. 109-121, 2002.

[10] M. Ciarletta and A. Scalia, "Some results in linear theory of thermomicrostretch elastic solids," Meccanica, vol. 39, no. 3, pp. 191-206, 2004.

[11] R. J. Knops and L. E. Payne, "Alternative spatial growth and decay for constrained motion in an elastic cylinder," Mathematics and Mechanics of Solids, vol. 10, no. 3, pp. 281-310, 2005.

[12] S. Chiriţă and M. Ciarletta, "Spatial behavior for some non-standard problems in linear thermoelasticity without energy dissipation," Journal of Mathematical Analysis and Applications, vol. 367, no. 1, pp. 58-68, 2010.

[13] E. Bulgariu, "Alternative spatial growth and decay estimates for constrained motion in an elastic cylinder with voids," Analele Stiintifice ale Universitatii Al. I. Cuza din Iasi-Matematica, vol. 58, 2011.

[14] R. Quintanilla and B. Straughan, "Energy bounds for some non-standard problems in thermoelasticity," Proceedings of the Royal Society A, vol. 461, no. 2056, pp. 1147-1162, 2005. 


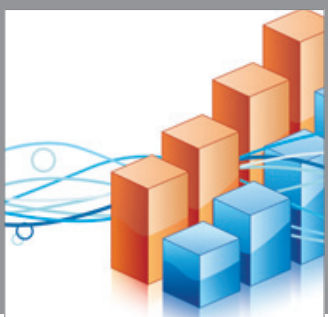

Advances in

Operations Research

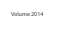

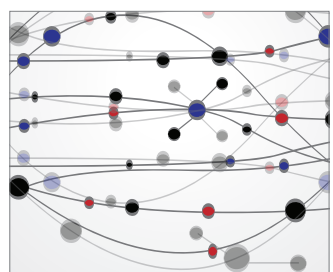

\section{The Scientific} World Journal
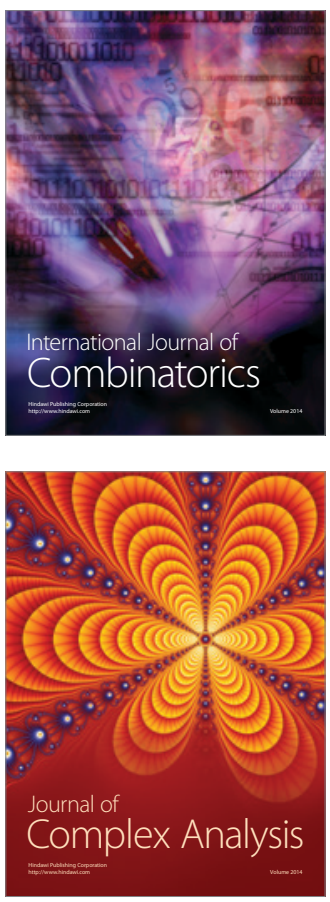

International Journal of

Mathematics and

Mathematical

Sciences
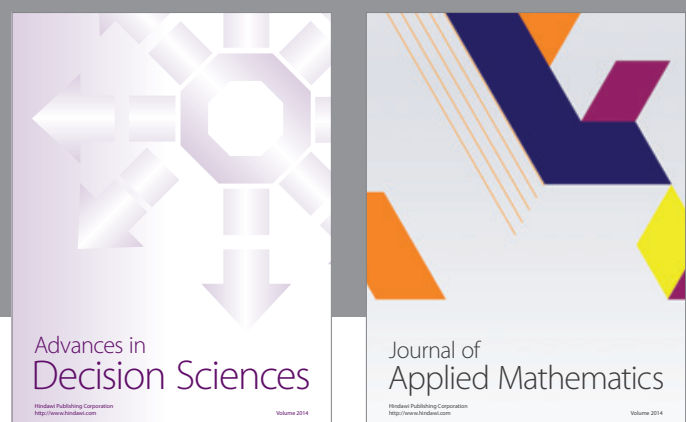

Journal of

Applied Mathematics
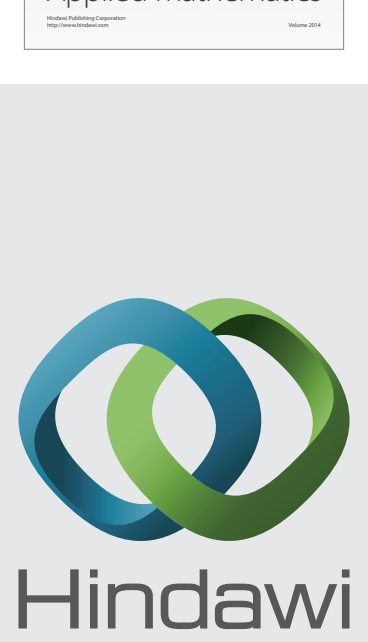

Submit your manuscripts at http://www.hindawi.com
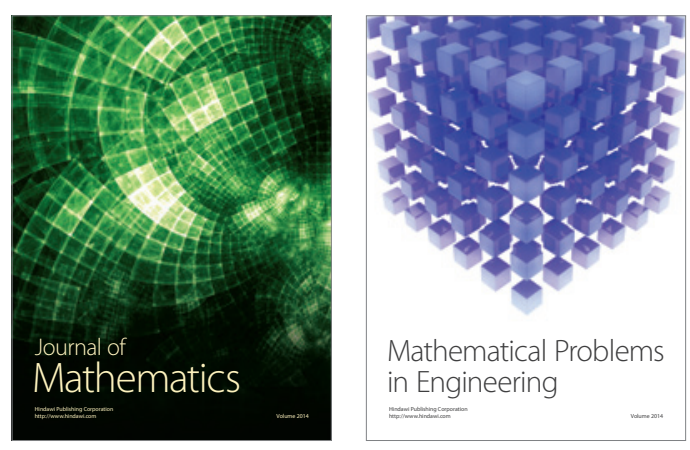

Mathematical Problems in Engineering
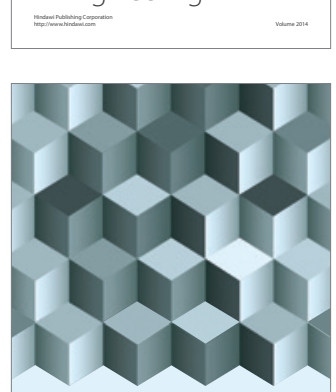

Journal of

Function Spaces
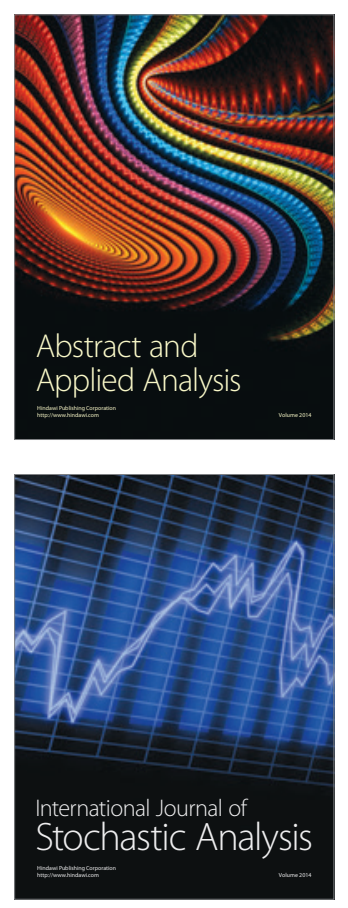

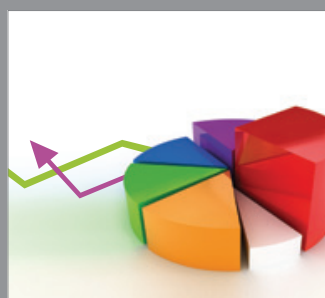

ournal of

Probability and Statistics

Promensencen
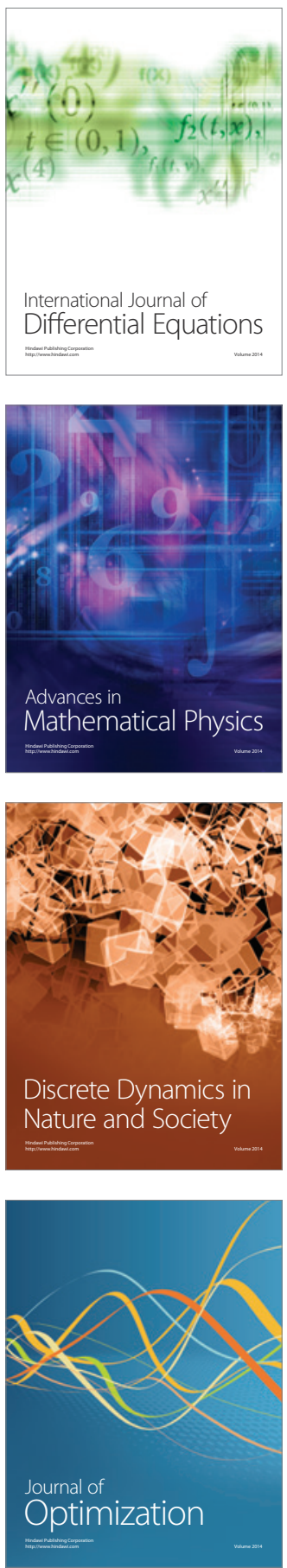